\title{
Diseño y Construcción de una Máquina de Soldadura de Fricción por Aglomeración
}

\author{
Oscar Francisco Sánchez-Femat ${ }^{1}$ (D), Miguel Fernando Delgado-Pamanes ${ }^{1}$ (D), Luis Arturo Reyes- Osorio ${ }^{2}$ (D), Miguel Ángel Suárez-Rosales ${ }^{3}$ (D), \\ José Federico Chávez-Alcala ${ }^{4}$ (D), Rafael Flores-Pinedo ${ }^{1}$ (i) , Josúe Daniel Becerra-Márquez ${ }^{1}$ (D) \\ ${ }^{1}$ Instituto Politécnico Nacional, Unidad Profesional Interdisciplinaria de Ingeniería Campus Zacatecas - UPIIZ, Profesional Genérica Ingeniería Mecatrónica \\ Zacatecas, México. \\ ${ }^{2}$ Universidad Autónoma de Nuevo León, Facultad de Ingeniería Mecánica y Eléctrica, CIIIA, Nuevo León, México. \\ ${ }^{3}$ Universidad Autónoma Metropolitana, Departamento de Materiales, Ciudad de México, México. \\ ${ }^{4}$ Instituto Politécnico Nacional, Escuela Superior de Ingeniería Química e Industrias Extractivas - ESIQIE, UPALM, Ciudad de México. México.
}

Como citar: Sánchez-Femat OF, Delgado-Pamanes MF, Reyes-Osorio LA, Suárez-Rosales MA, Chávez-Alcala JF, Flores-Pinedo R, et al. Diseño y construcción de una máquina de soldadura de fricción por aglomeración. Soldagem \& Inspeção. 2021;26:e2607. https://doi.org/10.1590/0104-9224/SI26.06.

\begin{abstract}
Resumen: Los procesos de soldadura por fricción son utilizados en áreas diversas tales como el sector naval, automotriz y aeroespacial. Estos procesos ofrecen una gran flexibilidad y eficiencia energética en la generación de uniones. La soldadura de fricción por aglomeración es un proceso de unión en estado sólido, en el que una herramienta giratoria opera a lo largo de una línea de unión entre dos piezas generando calor por fricción y agitación mecánica para formar la unión de soldadura. En este trabajo, se presenta el diseño y construcción de una máquina de soldadura de fricción por aglomeración, considerando las etapas: a) diseño de disco de soldadura, b) cálculo en la preparación de láminas, c) selección del motor - tornillo de avance y d) cálculo de la estructura de soporte. En la evaluación del equipo se desarrollaron uniones de soldadura en aleaciones ligeras de $\mathrm{Zn}-22 \% \mathrm{Al}-2 \% \mathrm{Cu}$ (zinalco) y Al A380 generando cordones de soldadura con un flujo continuo de material.
\end{abstract}

Palabras-clave: Soldadura de fricción por aglomeración; simulación; diseño.

\section{Design and Construction of a Novel Friction Crushing Welding Machine}

Abstract: Friction welding processes are used in diverse areas such as the naval, automotive, and aerospace sectors. These processes offer great flexibility and energy efficiency in the generation of joints. Friction crush welding is a solidstate joining process in which a rotating tool operates along a joint line between two parts generating frictional heat and mechanical agitation to form a joint. In this work, the design and construction of a friction crush welding machine is presented, the stages included: a) the welding disc design, b) calculus of sheets, c) selection of the motor-screw advancement and d) calculus of the support structure. In the evaluation of the equipment, weld joints of $\mathrm{Zn}-22 \% \mathrm{Al}-2 \%$ $\mathrm{Cu}$ (zinalco) and Al A380 alloy were developed presenting weld beads with a continuous material flow.

Key-words: Friction crush welding; analysis; design.

\section{Introducción}

El proceso de soldadura por fricción-agitación FSW (Friction Stir Welding) se desarrolló en 1991 en Cambridge, Reino Unido. El uso de FSW ha ganado un papel destacado en la producción de soldadura altamente integrada en fase sólida en aleaciones de aluminio [1]. Sin embargo, recientemente se han desarrollado investigaciones en materiales como aleaciones de cobre, magnesio, titanio, aceros inoxidables, compuestos de matriz metálica (MMCs), entre otros [2]. En el proceso de soldadura por fricción-agitación el movimiento giratorio de una herramienta a lo largo de una línea de unión entre dos piezas genera calor por fricción y agitación mecánica para formar una unión. La herramienta giratoria consiste en un hombro cilíndrico y un pin que se proyecta debajo del hombro. Durante la soldadura, el hombro genera fricción contra la superficie desarrollando gran parte del calor de fricción, mientras que el pin genera calor adicional al mezclar mecánicamente el metal a lo largo de las superficies. El pin posee una geometría diseñada para facilitar la acción de mezcla. Los parámetros de soldadura como carga axial, velocidad rotacional, velocidad de avance, entre otros, determinan las propiedades mecánicas de la unión. Las características de la unión como: metal base, tipo de junta y espesor permiten la identificación de parámetros en la optimización de las propiedades [3]. Una de las mayores ventajas de la técnica FSW es su potencial para refinar el grano en la zona de soldadura. El proceso FSW se ha tomado como referencia para estudiar nuevos métodos de unión por fricción de los cuales destacan el rotatorio, el lineal y el orbital, siendo categorizados de acuerdo con la naturaleza del movimiento de las superficies de las piezas a unir y al tipo de

Recibido em 15 Jun 2020. Aprobado 05 Ene. 2021

E-mail: mfdelgadop@ipn.mx (MFDP)

Este es un artículo publicado en acceso (Open Access) abierto bajo la licencia Creative Commons Attribution Non-Commercial, que permite su uso, distribución y reproducción en cualquier medio, sin restricciones siempre que sin fines comerciales y que el trabajo original sea debidamente citado. 
energía aplicado del proceso [4]. El proceso de soldadura por fricción es ampliamente utilizado para la unión materiales difíciles de soldar [5]. Sin embargo, el entendimiento profundo del proceso de fricción para materiales de alta resistencia es limitado [6]. En el proceso FSW los esfuerzos residuales y distorsiones asociadas se reducen notablemente debido a las menores temperaturas alcanzadas en comparación con los procesos de soldadura por fusión [7].

El proceso de soldadura de fricción por aglomeración "FCW" (por su acrónimo en inglés Friction Crush Welding) es un método nuevo, versátil y apropiado para soldar láminas delgadas [8]. El principio de funcionamiento es similar al proceso FSW, el movimiento relativo de fricción, en contraste con FSW, se logra por medio de un disco giratorio y con una velocidad unidireccional entre el disco y los bordes de las láminas ver Figura 1. El diseño del disco cuenta con un perfil circunferencial específico, que al contacto con los bordes de las láminas produce un calentamiento por la fricción y debido a la fuerza ejercida del disco sobre los bordes de la lámina forzando así cierto volumen a mezclarse para crear una unión.

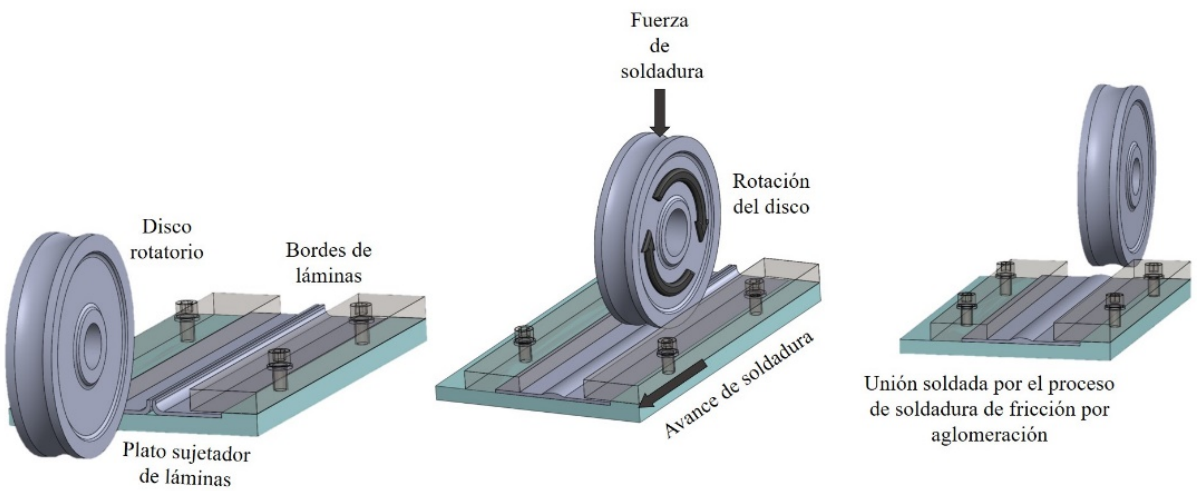

Figura 1. Esquemático del proceso de soldadura de fricción por aglomeración (FCW).

Dentro de los principales estudios realizados en FCW destacan el trabajo realizado por Besler et al [8] en el que utilizando fricción por aglomeración desarrolló uniones de láminas de metal de aluminio, acero y cobre sin material de aporte [8]. La calidad de la soldadura se midió en términos de resistencia de la unión y análisis microestructural. Brar and Jamwal [9] desarrollaron uniones de Al 6061 mediante fricción por aglomeración, donde los parámetros de proceso de salida se midieron en términos de carga a la ruptura respecto al perfil de la herramienta, la rotación de la herramienta giratoria (rpm) y la velocidad de avance ( $\mathrm{mm} / \mathrm{min}$ ). Los resultados experimentales arrojaron que se puede lograr la máxima fuerza de unión cuando existe un aumento en la velocidad de la herramienta giratoria y la velocidad de avance [9]. Aunque existen trabajos recientes enfocados al proceso de soldadura de fricción por aglomeración, la literatura disponible es limitada siendo de interés el desarrollo de más investigaciones del proceso FCW en la unión de aleaciones ligeras.

\section{Metodología}

\subsection{Requisitos del diseño}

En el diseño de una máquina de soldadura de fricción por aglomeración se incluyen el diseño de disco de soldadura, cálculos en la preparación de láminas, selección del motor y tornillo de avance. Existen componentes básicos de importancia, entre ellos destaca el sistema principal del husillo (donde se coloca el disco rotatorio de soldadura), este sistema proporciona una potencia constante en un amplio rango de velocidades de rotación (de 0 a $24.000 \mathrm{rpm}$ ) y velocidad de desplazamiento lineal (de 0 a $10.000 \mathrm{~mm} / \mathrm{min}$ ). Para una selección adecuada de los componentes mecánicos y eléctricos se deberá calcular las fuerzas producidas durante el proceso de FCW. La Figura 2, muestra un diagrama de las principales etapas en el diseño y construcción de una máquina de soldadura de fricción por aglomeración.

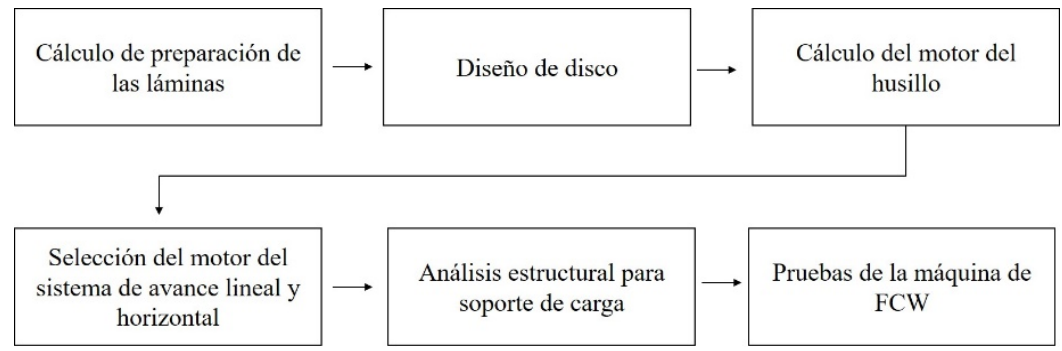

Figura 2. Diagrama de las principales etapas en el diseño y construcción de una máquina de soldadura de fricción por aglomeración. 


\subsection{Cálculos de preparación de las láminas}

Las láminas requieren de una preparación especial (ver Figura 3), esta preparación consiste en un doblez de borde como material adicional, la función del material adicional es aportar el 86,5\% de material en la zona de soldadura (zona deformación plástica) y el $13,5 \%$ para formar la corona de soldadura. Para calcular el material adicional se considera un volumen $V_{3}$ definido mediante la ecuación 1:

$V_{3}=2 A t^{*} L$

Donde $A$ representa la altura del material adicional, $t$ el espesor de la lámina y $L$ es la longitud de la lámina. Un aspecto importante en la preparación es la separación entre los bordes de las láminas $(G)$ que permite el flujo de material adicional desde los bordes de láminas hacia la zona de unión durante el proceso de FCW.

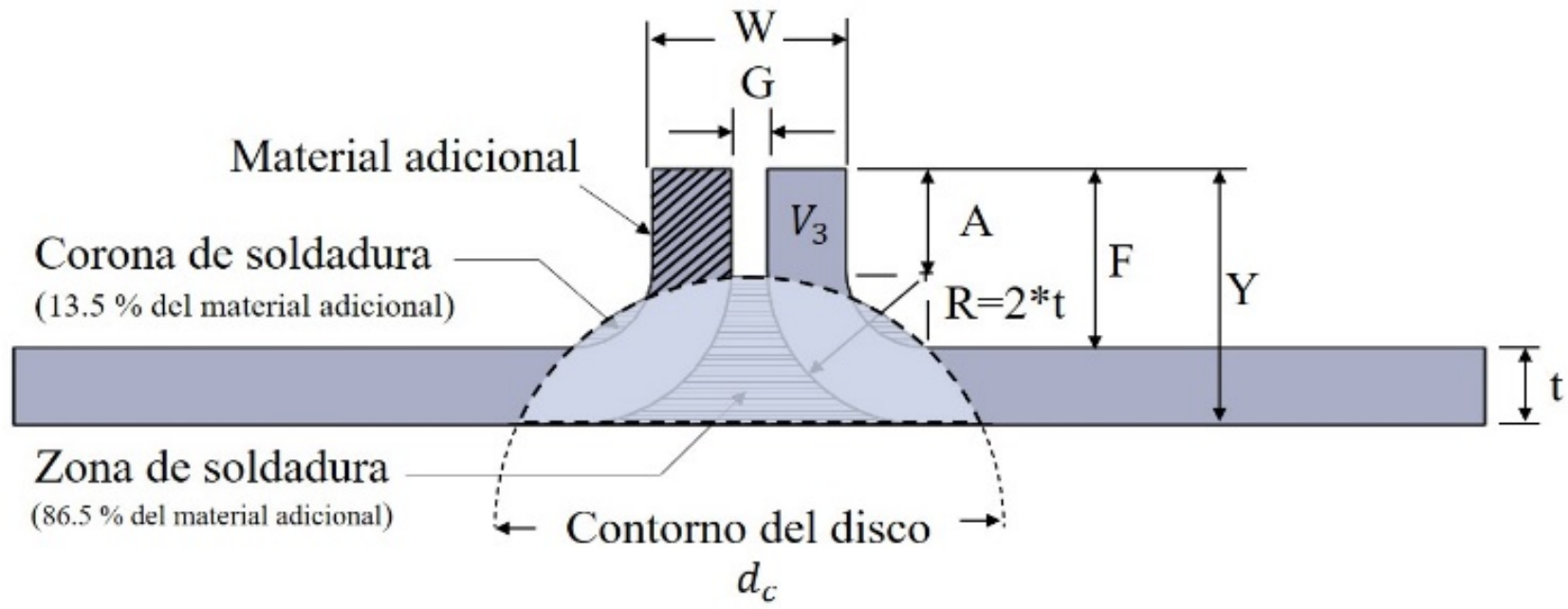

Figura 3. Requerimientos de preparación de las láminas.

La altura de material adicional $A$ se relaciona con el espesor y la separación entre bordes de las láminas mediante la ecuación 2:

$A=G+t(4-\pi)$

La altura externa del borde de lámina $Y$ se relaciona con la altura del material adicional y el radio externo de borde $R$ mediante la ecuación 3:

$Y=A+R$

Las dimensiones de las láminas son $100 \mathrm{~mm}$ de longitud, $20 \mathrm{~mm}$ de ancho y 2,2 mm de espesor, se propone una separación entre bordes de $1 \mathrm{~mm}$, la altura del material adicional es $A=1 \mathrm{~mm}+2,2 \mathrm{~mm}(4-\pi)=2,88 \mathrm{~mm}$ y la altura externa del borde de lámina $Y=2,88 \mathrm{~mm}+4,4 \mathrm{~mm}=7,28 \mathrm{~mm}$.

\subsection{Diseño del disco}

El diseño del disco de soldadura se describe mediante las dos etapas del proceso; en la primera etapa el perfil o contorno del disco $d_{c}$, que al contacto con los bordes de las láminas produce un calentamiento por la fricción y debido a la fuerza ejercida del disco sobre los bordes de la lámina se genera deformación plástica en las láminas. En la segunda etapa el hombro del disco $S$ aplica presión en el material generando deformación plástica en una capa relativamente delgada debajo de la superficie del hombro, como se representa en Figura 4. 


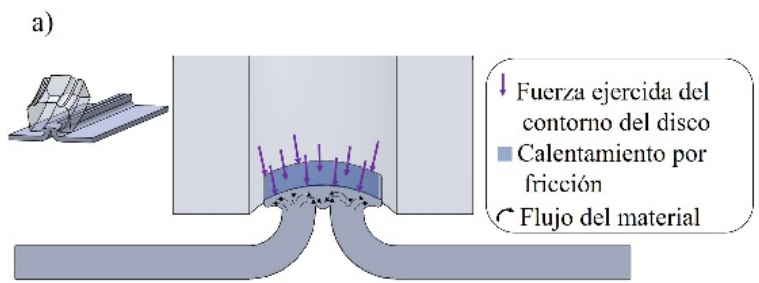

b)
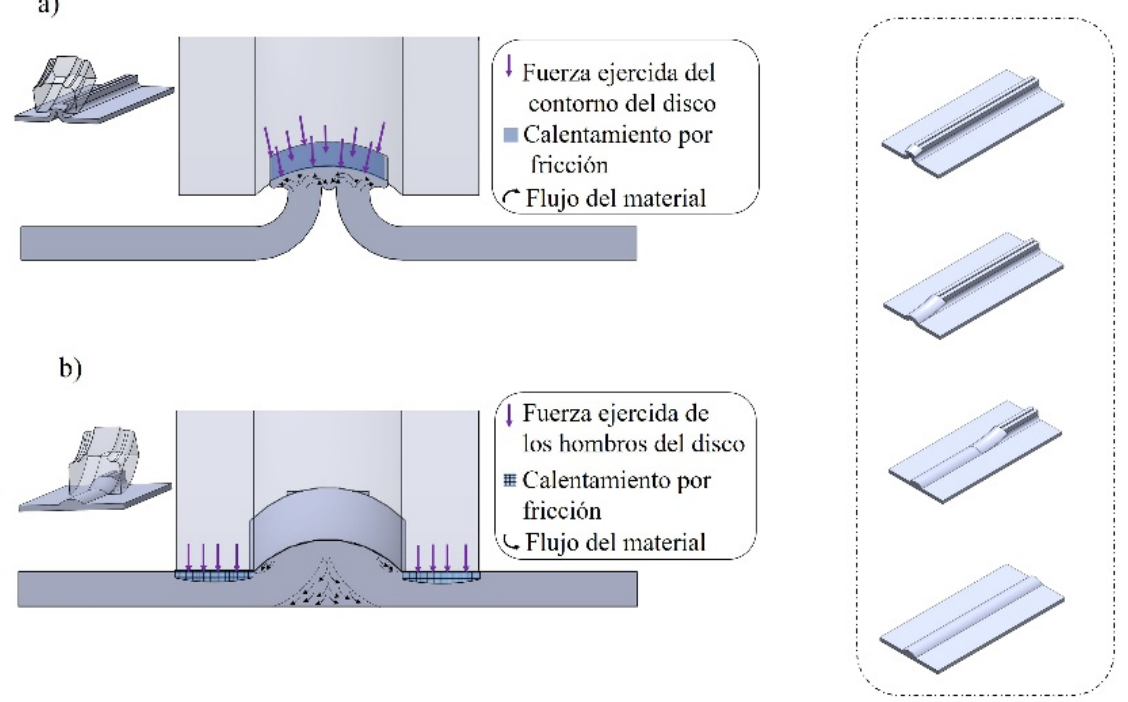

Figura 4. Etapas de acción de soldadura durante el proceso FCW. a) primera etapa contacto entre el disco y los bordes de las láminas y b) segunda etapa contacto de los hombros del disco en la superficie de las láminas.

La geometría del disco de soldadura se muestra en la Figura 5. El diámetro del contorno del disco se obtiene mediante la ecuación 4:

$d_{c}=\sqrt{2} *(4 * t+G)$

El ancho del contorno del disco se expresa en la ecuación 5:

$W_{C}=2 * R+G$

La longitud de contacto entre los materiales a soldar $L_{p}$ y el disco de soldadura se puede estimar con la ecuación 6 [10]:

$L_{P}=\sqrt{R_{D}(A)}$

Donde $R_{D}$ es el radio de contacto interno del disco.
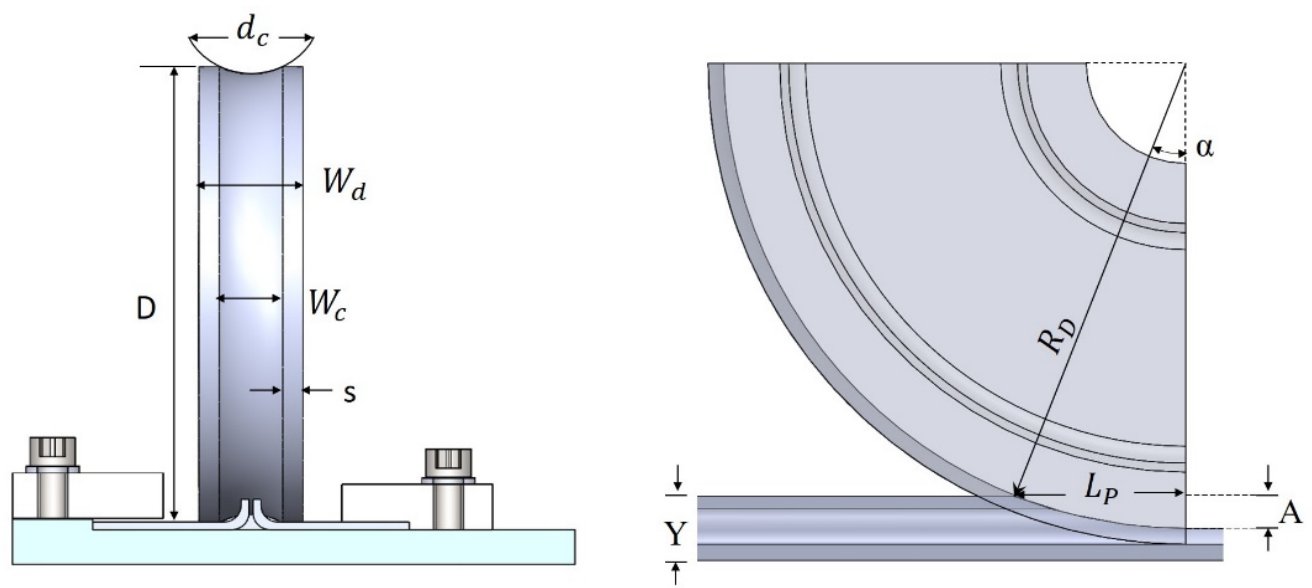

Figura 5. Geometría del disco de soldadura y longitud de contacto del disco.

Se considera una disco de $120 \mathrm{~mm}$ de diámetro y $5 \mathrm{~mm}$ de hombro para obtener los valores correspondientes a la geometría del disco tal y como se muestran en la Tabla 1. 
Tabla 1. Parámetros en el diseño de disco.

\begin{tabular}{ccc}
\hline Característica & Desarrollo & Valor (mm) \\
Diámetro del contorno del disco, $d_{c}$ & $d_{c}=\sqrt{2} *(4 * 2,2 \mathrm{~mm}+1 \mathrm{~mm})$ & 13,85 \\
Ancho del contorno del disco, $W_{C}$ & $W_{C}=2 * 4,4+1$ & 9,8 \\
$\begin{array}{c}\text { Longitud de contacto entre los materiales a } \\
\text { soldary el disco, } L_{P}\end{array}$ & $L_{P}=\sqrt{57,8 \mathrm{~mm}(2,88 \mathrm{~mm})}$ & 12,90 \\
\hline
\end{tabular}

De manera adicional, se realizaron estudios en Aluminio A-380 para encontrar la óptima geometría de disco con relación entre esfuerzo y deformación. Se llevaron a cabo simulaciones con las geometrías definidas en la Tabla 2. El objetivo de este estudio fue observar el efecto de la geometría del disco en el esfuerzo necesario para deformar el material de la unión.

Tabla 2. Características del disco rotatorio.

\begin{tabular}{ccccccc}
\hline \multirow{2}{*}{$\begin{array}{c}\text { Característica geométrica } \\
(\mathbf{m m})\end{array}$} & Calculado & \multicolumn{5}{c}{ Número de disco para soldadura } \\
\cline { 2 - 7 } & & D1 & D2 & D3 & D4 & D5 \\
\cline { 2 - 7 } Radio de curvatura, $R_{C}$ & 6,925 & 7,93 & 19,30 & 20,00 & 22,00 & 10,00 \\
Hombro, S & 5,00 & 5,00 & 0,50 & 2,80 & 2,80 & 5,00 \\
Radio del disco, $R_{D}$ & 60,00 & 60,00 & 60,00 & 40,00 & 40,00 & 40,00 \\
Espesor, $W_{D}$ & 19,80 & 20,00 & 20,00 & 20,00 & 25,00 & 25,00 \\
\hline
\end{tabular}

\subsection{Cálculo del motor del husillo}

El husillo debe proporcionar la energía necesaria para que el disco de soldadura pueda deformar plásticamente los bordes de las láminas, a fin de producir un cordón de soldadura. Para la realización de los cálculos se utiliza el principio de laminación, expresados desde la ecuación 7 a la 9.

$$
\begin{aligned}
& \boldsymbol{F}=\overline{\boldsymbol{Y}}_{\boldsymbol{F}} * \boldsymbol{W} * \boldsymbol{L}_{\boldsymbol{P}} \\
& \overline{Y_{F}}=\frac{K \epsilon^{n}}{1+n} \\
& \in=\ln \left(\frac{Y}{R}\right)
\end{aligned}
$$

Donde $\bar{Y}_{F}$ es el esfuerzo de cedencia promedio, $W$ es la distancia entre los bordes de las láminas, $K$ es el coeficiente de resistencia, $\epsilon$ es máximo valor de deformación durante el proceso y $n$ es el coeficiente de endurecimiento del material [11].

El torque y la potencia se calculan mediante las ecuaciones 10 y 11 :

$T=0,5^{*} F^{*} L_{P}$

$P=\frac{2 * \pi * T^{*} N}{60.000}$

Donde $T$ es el torque del motor, $N$ es la velocidad en $\mathrm{rpm}$ del rodillo.

Se considera un valor de $W$ de 5,40 mm, la longitud de contacto del disco $L_{p}$ de $12,90 \mathrm{~mm}$, valor de cedencia de $205 \mathrm{MPa}$, coeficiente de endurecimiento por deformación 0,20 y las revoluciones por minuto del disco de 1.000.

Las características del husillo están definidas por los valores mostrados en la Tabla 3.

Para validar los resultados de la fuerza, se realizó un estudio de elemento finito mediante el software Ansys. En la Figura 6 , se observa la deformación del metal base que se produce al realizar la soldadura, así como el esfuerzo necesario para lograr la aglomeración del metal base. 
Tabla 3. Características y cálculos del motor del husillo.

\begin{tabular}{|c|c|c|}
\hline Característica & Desarrollo & Valor \\
\hline Máximo valor de deformación, $\epsilon$ & $\in=\ln \left(\frac{7,28 \mathrm{~mm}}{4,4 \mathrm{~mm}}\right)$ & 0,503 \\
\hline Esfuerzo de cedencia promedio, $\bar{Y}_{F}$ & $\overline{Y_{F}}=\frac{205 M P a^{*} 0,504^{0,20}}{1+0,20}$ & $148,95 \mathrm{MPa}$ \\
\hline Fuerza, $F$ & $F=148,95 M^{2} a^{*} 0,0054 m^{*} 0,0129 m$ & $10,375 \mathrm{kN}$ \\
\hline Torque, $T$ & $T=0,5^{*} 10,375 k N^{*} 0,0129 \mathrm{~m}$ & $66,918 \mathrm{Nm}$ \\
\hline Potencia, $P$ & $P=\frac{2 * \pi * 66,918 \mathrm{Nm}^{*} 1.000 \mathrm{rpm}}{60.000}$ & $7,01 \mathrm{~kW}$ \\
\hline
\end{tabular}
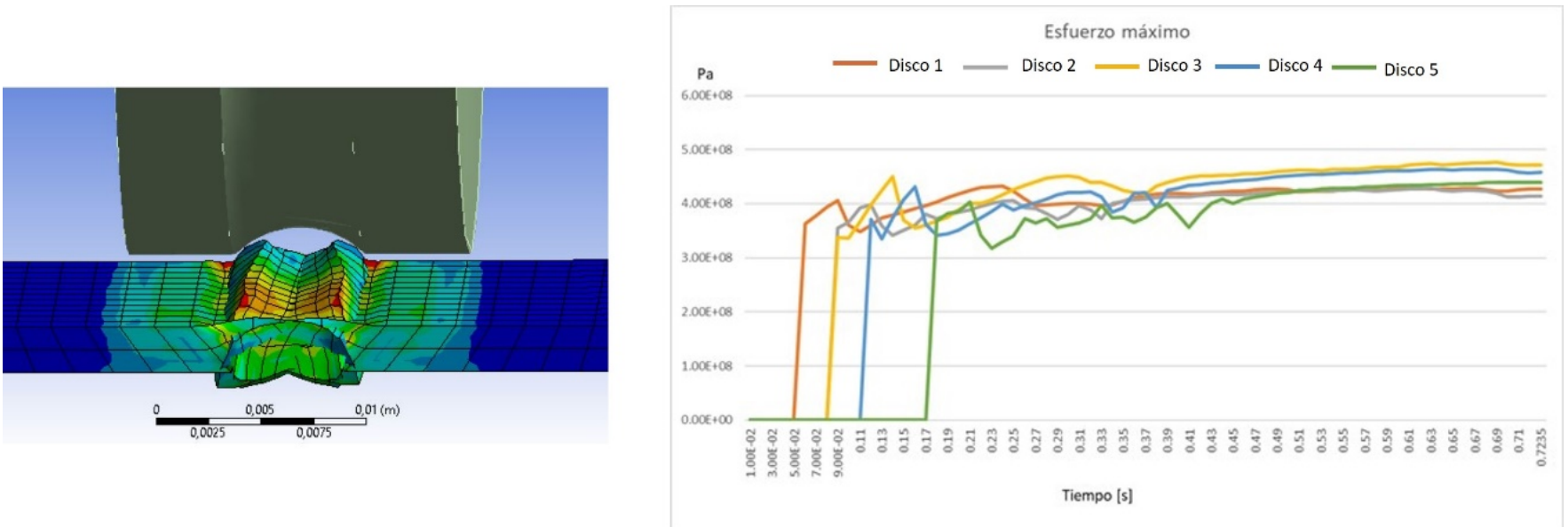

Figura 6. Simulación de esfuerzo equivalente de Von Mises, necesario para producir la deformación de los bordes de las láminas utilizando las diferentes geometrías de discos de soldadura.

Se seleccionó un motor de husillo de 7,5 kW, con un rango de velocidad de 0 a $24.000 \mathrm{rpm}$. En la Figura 7, se muestra el sistema del rotor donde se ubica la herramienta giratoria (disco de soldadura).

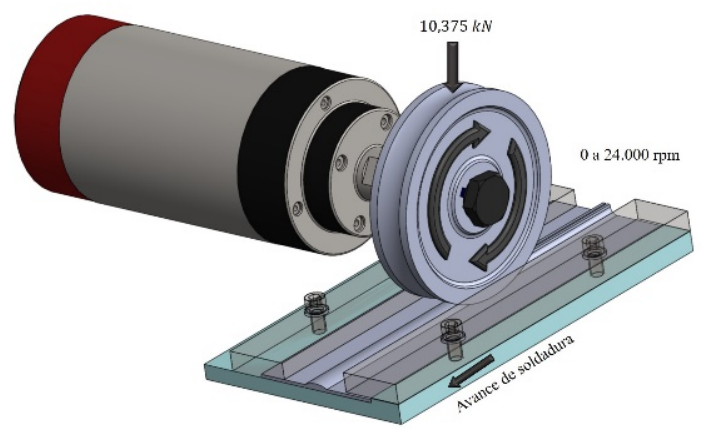

Figura 7. Sistema del husillo de 7,5 kW con un rango de velocidad 0 a $24.000 \mathrm{rpm}$ donde se coloca la herramienta giratoria.

\subsection{Selección del motor del sistema de avance lineal y horizontal}

El diseño del sistema de avance lineal y horizontal de soldadura está conformado de rodamientos lineales, guías lineales, tornillo de bola y motor eléctrico, estos elementos transforman el movimiento rotatorio del motor a un movimiento lineal, ver Figura 8. a) El sistema de avance lineal requiere una velocidad de avance de soldadura en un rango de 0 a $10.000 \mathrm{~mm} / \mathrm{min}$. El torque necesario para mover el sistema de avance es de $2,72 \mathrm{Nm}$ b) El diseño del sistema del movimiento horizontal que controla la velocidad ascendente y descendente en un rango de 0 a $100 \mathrm{~mm} / \mathrm{min}$. El torque requerido para mover este sistema es de 40 $\mathrm{Nm}$. El torque de cada sistema se obtuvo con Solidworks motion. Se propuso un tornillo de $20 \mathrm{~mm}$ de diámetro, sus características se muestran en la Tabla 4. 
Tabla 4. Características del tornillo.

$\begin{array}{cc}\text { Característica } & \text { Valor }(\mathbf{m m}) \\ \text { Diámetro, } \varnothing & 20,00 \\ \text { Paso de rosca, } \mathrm{P} & 2,00 \\ \text { Diámetro de rosca, } \emptyset_{\mathrm{r}} & 28,64 \\ \text { Diámetro de paso, } \emptyset_{\mathrm{p}} & 19,18 \\ \text { Diámetro de medio, } \emptyset_{\mathrm{m}} & 19,00\end{array}$

Nota: Torque necesario para la carga $\alpha=30^{\circ}$ y coeficiente de rozamiento tuerca tornillo $\mu_{0}=0,15$.

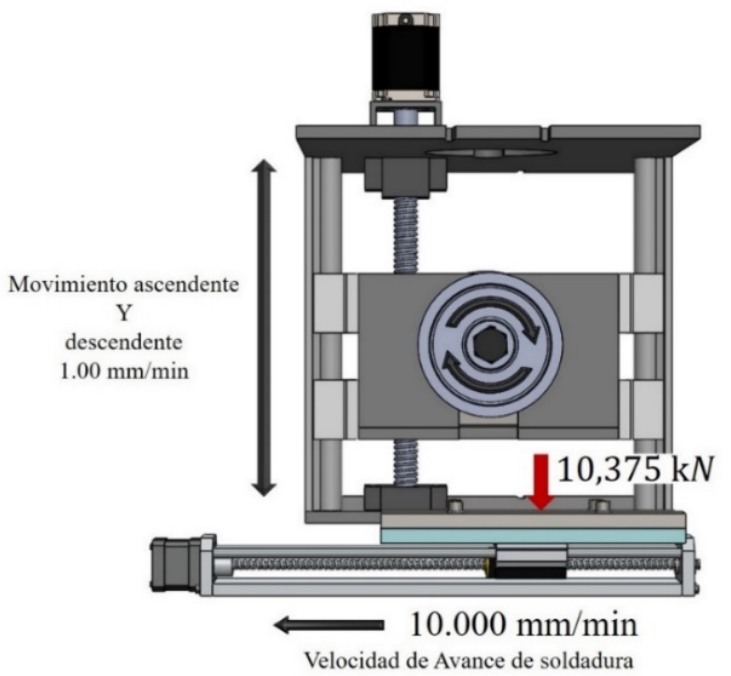

Figura 8. Sistema de movimiento del motor y tornillo de avance.

La velocidad de desplazamiento se obtiene mediante la ecuación 12:

$V=\frac{P^{*} N}{60}$

Donde $V$ es la velocidad de desplazamiento $(\mathrm{m} / \mathrm{s}), P$ es paso de tornillo y $N$ es la velocidad en rpm.

Las características del sistema de avance lineal y horizontal se muestran en la Tabla 5 y Tabla 6 respectivamente.

Tabla 5. Cálculos del motor del sistema de avance lineal.

\begin{tabular}{ccc}
\hline Característica & Desarrollo & Valor \\
Revoluciones por minutos, $\mathrm{N}$ & $N=\frac{60(0,166 \mathrm{~m} / \mathrm{s})}{0,002}$ & $5000 \mathrm{rpm}$ \\
Potencia requerida, $\mathrm{P}$ & $P=\frac{2^{*} \pi^{*} 2,723 \mathrm{~N}^{*} \mathrm{~m}^{*} 5.000 \mathrm{rpm}}{60.000}$ & $1,42 \mathrm{~kW}$
\end{tabular}

Tabla 6. Cálculos del motor del sistema de avance horizontal.

\begin{tabular}{ccc}
\hline Característica & Desarrollo & Valor \\
Revoluciones por minutos, $\mathrm{N}$ & $N=\frac{60(0,0166667 \mathrm{~m} / \mathrm{s})}{0,002}$ & $500 \mathrm{rpm}$ \\
Potencia requerida, $\mathrm{P}$ & $P=\frac{2 * \pi^{*} 40 \mathrm{~N}^{*} \mathrm{~m}^{*} 500 \mathrm{rpm}}{60.000}$ & $2,09 \mathrm{~kW}$ \\
\hline
\end{tabular}


La selección de los motores para la máquina de soldadura FCW, es la siguiente un motor de husillo de 7,5 kW con un rango de velocidad de 0 a $24.000 \mathrm{rpm}$, en el sistema de avance de soldadura y en el sistema de elevación se utilizaron tornillos de potencia M20x2,5 de 25 mm y motores NEMA23 y NEMA34 respectivamente.

\subsection{Diseño de la estructura de soporte}

En el diseño de la estructura principal que soporta el conjunto de fuerzas generadas durante el proceso de FCW se propone el uso de vigas de perfil IR y canal CPS (CE), sus propiedades mecánicas se muestran en Tabla 7. Para este análisis se realizaron simulaciones estructurales de la máquina FCW, se propuso una fuerza de $212,33 \times 106 \mathrm{~N} / \mathrm{m} 2$. El resultado del análisis de esfuerzo y factor de seguridad se muestran en la Figura 9. La estructura soporta las fuerzas necesarias para el proceso debido a que el factor de seguridad mínimo es igual a 6,15 y los resultados mostrados por el cálculo de esfuerzo Von Mises presentan un valor global de 0 a 4,059 x $107 \mathrm{~N} / \mathrm{m} 2$ y un límite elástico de 2,5 x $108 \mathrm{~N} / \mathrm{m} 2$. Estos resultados confirman que el uso de la estructura para el proceso de soldadura es adecuado y seguro de utilizar.

Tabla 7. Propiedades mecánicas del acero A36.

\begin{tabular}{cc} 
Propiedad & Valor (N/m2) \\
Módulo de Young, $E$ & $2 \times 1011$ \\
Esfuerzo Último de Tensión, $S_{u t}$ & $40,00 \times 106$ \\
Esfuerzo de Cedencia, $S_{y}$ & $250,00 \times 106$ \\
\hline
\end{tabular}

a)

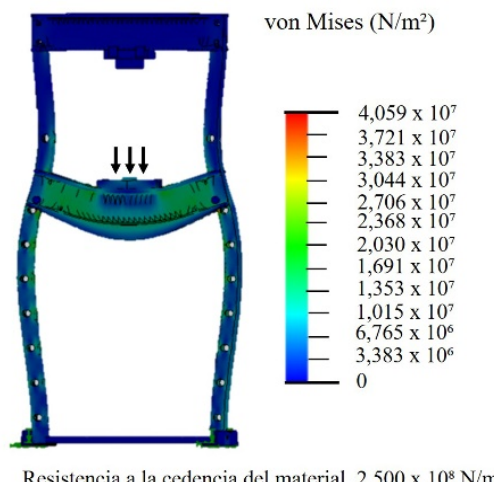

b)

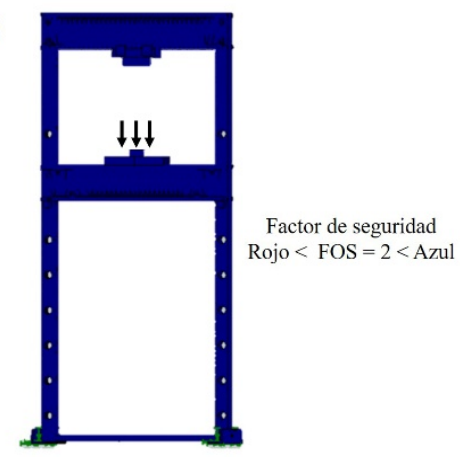

Figura 9. Resultados de las simulaciones a) esfuerzo Von Mises y b) factor de seguridad en la estructura.

El diseño final de la máquina de soldadura de fricción por aglomeración se muestra en la Figura 10, este equipo cumple con los requerimientos necesarios del proceso de FCW de acuerdo con los cálculos y simulaciones realizadas.
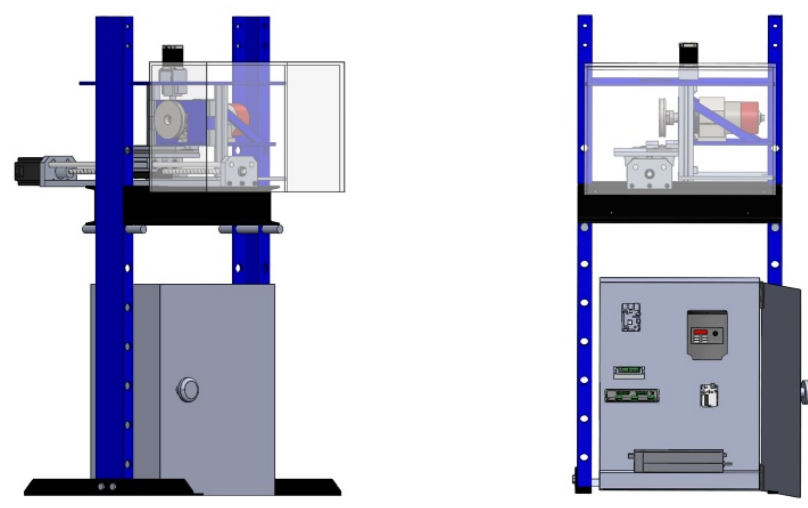

Figura 10. Diseño final de la máquina de soldadura de fricción por aglomeración.

\section{Resultados}

Una vez adquiridos todos componentes y manufacturado las piezas de la máquina de soldadura FCW, se ensambló tal como se muestra en Figura 11. 

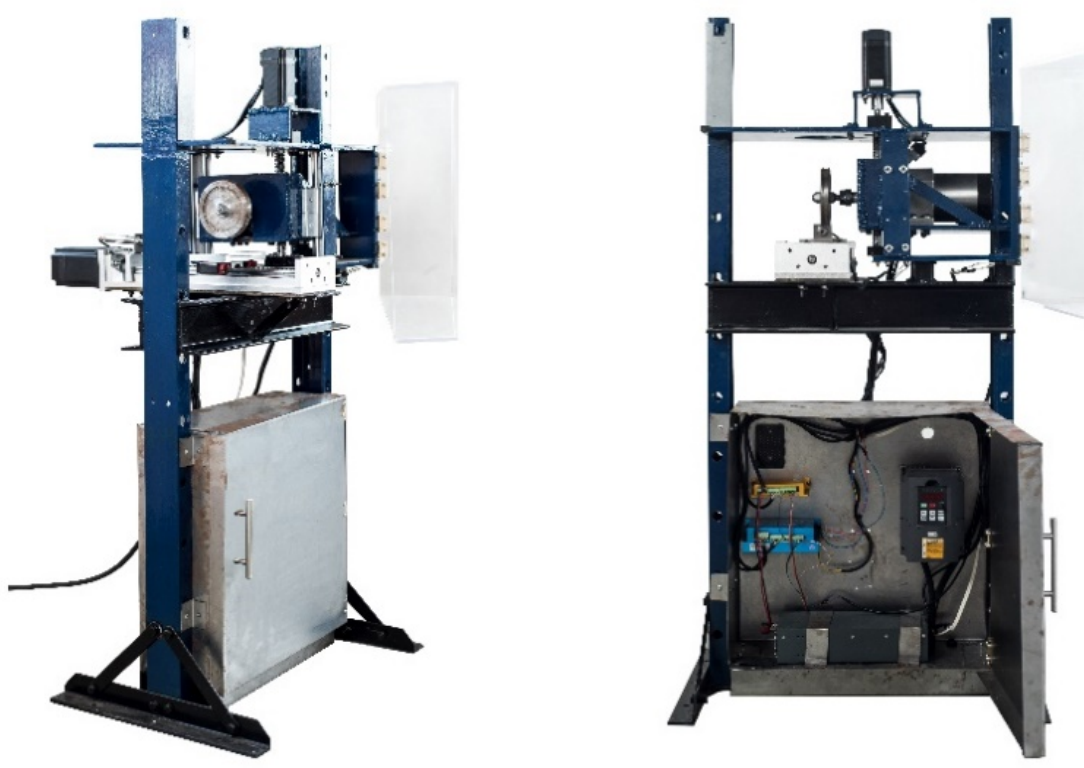

Figura 11. Construcción de la Máquina de soldadura de Fricción por aglomeración.

La Figura 12, muestra el funcionamiento de la máquina de soldadura de fricción por aglomeración, en la Figura 12a inicia el proceso de desplazamiento horizontal descendente y el encendido del sistema del husillo (de 15.000 y 18.000 rpm), en la Figura $12 \mathrm{~b}$ se muestra el desplazamiento lineal de $75 \mathrm{~mm} / \mathrm{min}$ (velocidad de $30 \mathrm{rpm}$ del motor nema), en esta etapa inicia el proceso de soldadura FCW, finalmente en la Figura 12c se muestra el cordón de soldadura FCW.

a)

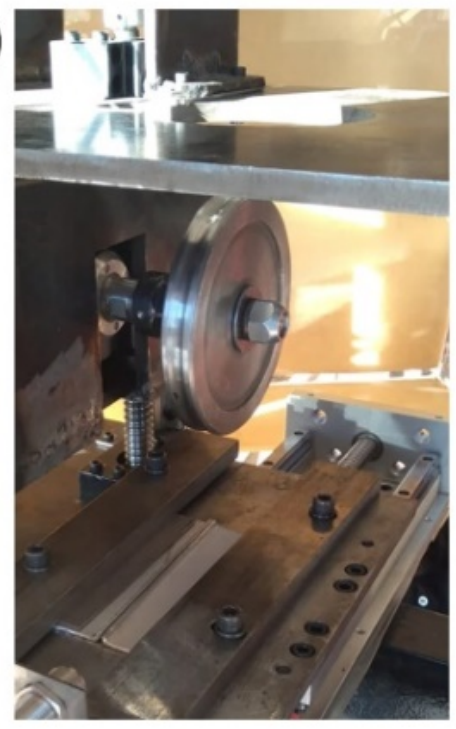

b)
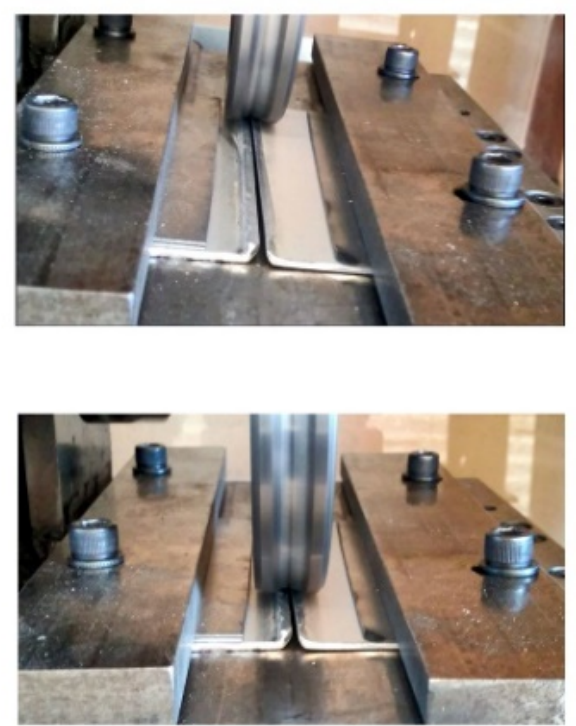

c)

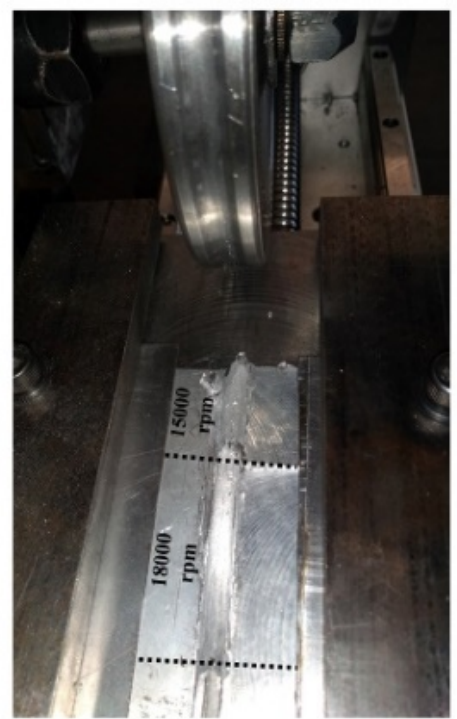

Figura 12. Funcionamiento de la máquina de soldadura de FCW.

La Figura 13, muestra las uniones desarrolladas por el proceso FCW. El inciso a) muestra el inicio del proceso de soldadura FCW, donde se aprecia la deformación de los bordes de las láminas. El inciso b), muestra la formación del cordón de soldadura que obtiene la forma del contorno del disco de soldadura. El inciso c) y el inciso d), muestran una unión de soldadura realizada a $15.000 \mathrm{rpm}$. El inciso d) muestra el flujo del material en la trayectoria de unión (parte posterior de la unión). El inciso e) y el inciso f) muestran una unión realizada a $18.000 \mathrm{rpm}$. A través de los resultados de las uniones soldadas, se demostró el funcionamiento y operabilidad del equipo de soldadura FCW, resultando en uniones que físicamente se observaron de buena calidad, sin embargo, es necesario realizar más estudios para verificar la calidad de las uniones. El objetivo del presente trabajo es aportar una metodología de diseño y construcción de un equipo de soldadura FCW en unión de materiales ligeros, siendo referencia para posteriores trabajos de la comunidad científica y tecnológica del área de soldadura en estado sólido. 
a)

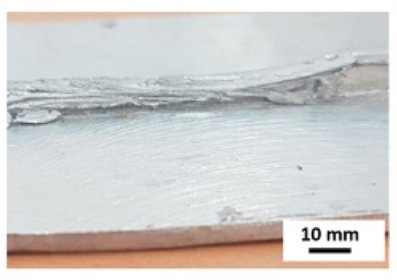

c)

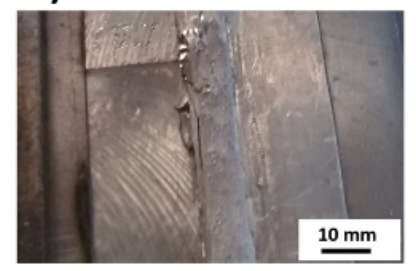

e)

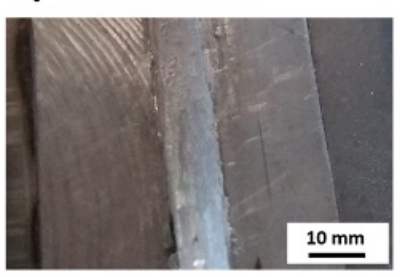

b)

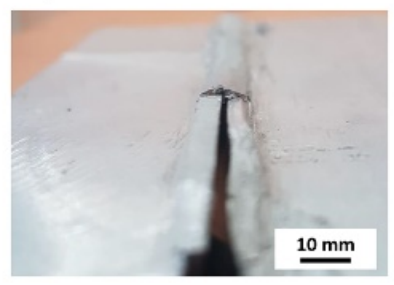

d)

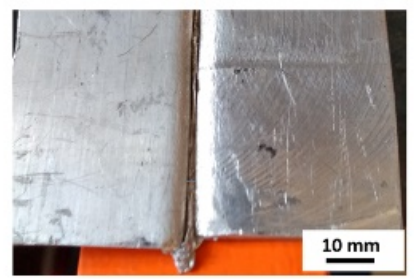

f)

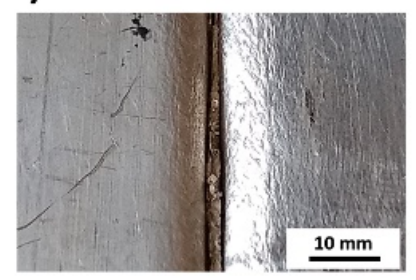

Figura 13. Uniones desarrolladas mediante soldadura de FCW.

\section{Conclusiones}

En este trabajo se desarrolló el diseño y construcción de una máquina de soldadura de fricción por aglomeración, la cual cumple con los requerimientos necesarios del proceso de FCW en base a los cálculos y simulaciones realizadas en el presente estudio. Se desarrollaron uniones de soldadura en las aleaciones ligeras generando cordones de soldadura con un flujo continuo de material. Se realizaron simulaciones de la estructura de la máquina FCW, donde se consideró una fuerza de $212,33 \times 106 \mathrm{~N} / \mathrm{m} 2$. Los resultados del análisis de esfuerzo y factor de seguridad de la estructura permiten el soporte de las fuerzas necesarias para el proceso, obtenidas de cálculos para el diseño, resultando una estructura para el proceso de soldadura adecuada y segura de utilizar.

Con este nuevo proceso se permitirá realizar nuevas investigaciones en el área de soldadura en estado sólido, se podrá evaluar el efecto de los parámetros primarios (revoluciones por minuto del disco y velocidad de avance de soldadura) y parámetros secundarios (geometría del disco de soldadura) del proceso FCW en la calidad de soldadura en uniones de materiales similares y disimiles.

\section{Referencias}

[1] Chai P, Hu W, Ji S, Ai X, Lv Z, Song Q. Refill friction stir spot welding dissimilar Al/Mg Alloys. Journal of Materials Engineering and Performance. 2019;28(10):6174-6181. http://dx.doi.org/10.1007/s11665-019-04359-7.

[2] Prater T. Friction stir welding of metal matrix composites for use in aerospace structures. Acta Astronautica. 2014;93:366-373, 373. http://dx.doi.org/10.1016/j.actaastro.2013.07.023.

[3] Álvarez Villar HN, Fierro V, Tufaro LN, Svoboda HG, Ansaldi AF, Gabarain RS et al., Estudio de propiedades mecánicas locales de uniones soldadas por Fricción Agitación (FSW) en AA6061-T6 mediante Small Punch Test (SPT). Revista Materia. 2018;23(2):1-11.

[4] Merino Hernández D. Soldadura de ejes por fricción, [tesis]. Barcelona, Espanha: Universitat Politècnica de Catalunya. Departament d'Enginyeria Mecànica; 2019.

[5] Trimble D, O'Donnell GE, Monaghan J. Characterisation of tool shape and rotational speed for increased speed during friction stir welding of AA2024-T3. Journal of Manufacturing Processes. 2015;17:141-150. http://dx.doi.org/10.1016/j.jmapro.2014.08.007.

[6] Toumpis A, Galloway A, Cater S, McPherson N. Development of a process envelope for Friction Stir Welding of DH36 Steel: A step change. Materials \& Design. 2014;62:64-75. http://dx.doi.org/10.1016/j.matdes.2014.04.066. 
[7] Zhang Y, Sato YS, Kokawa H, Park SHC, Hirano S. Microstructural characteristics and mechanical properties of Ti-6Al-4V Friction Stir Welds. Materials Science and Engineering A. 2008;485(1-2):448-455. http://dx.doi.org/10.1016/j.msea.2007.08.051.

[8] Besler F, Schindele P, Grant R, Stegmüller M. Friction crush welding of aluminium, copper and steel sheetmetals with flanged edges. Journal of Materials Processing Technology. 2016;234:72-83. http://dx.doi.org/10.1016/j.jmatprotec.2016.03.012.

[9] GS Brar, AS Jamwal. Friction crush Welding of Aluminium Alloy 6061 t-6. IJAMR. 2017;9(2):101-104.

[10] Jácome R, Giovanny E, Anaguano G, Darío A. Rediseño y optimización de laminadora de aluminio del laboratorio de metalografía de la facultad de ingeniería mecánica de la escuela Politécnica Nacional. Quito: Escuela Politécnica Nacional; 2016.

[11] Groover Mikell P. Fundamentos de manufactura moderna. 3rd ed. [s.I.]: Mc Graw Hill; 2007. 